\title{
THE ČECH COHOMOLOGY OF MOVABLE AND $n$-MOVABLE SPACES
}

\author{
BY \\ JAMES KEESLING $\left({ }^{1}\right)$
}

\begin{abstract}
In this paper the Čech cohomology of movable and $n \cdot$ movable spaces is studied. Let $X$ be a space and let $\check{H}^{k}(X)$ denote the $k$-dimensional Čch cohomology of $X$ with integer coefficients based on the numerable covers of $X$. Then if $X$ is movable, there is a subgroup $E$ of $\check{H}^{k}(X)$ which is the union of all the algebraically compact subgroups of $\check{H}^{k}(X)$. Furthermore, $\check{H}^{k}(X) / E$ is an $\kappa_{1}$-free abelian group. If $X$ is an $n$-movable space, then it is shown that this structure holds for $\check{H}^{k}(X)$ for $0<k<n$ and may be false for $k>n+1$. If $X$ is an $\mathrm{LC}^{n-1}$ paracompactum, then $X$ is known to be $n$-movable. However, in this case and in the case that $X$ is an $\mathrm{LC}^{n-1}$ compactum a stronger structure theorem is proved for $\check{H}^{k}(X)$ for $0<k<n-1$ than that stated above. Using these results examples are given of $n$-movable continua that are not shape equivalent to any $L^{n-1}$ paracompactum.
\end{abstract}

Introduction. In [1] Borsuk introduced the shape invariant notion of movability for compact metric spaces. In [13] Mardesić and Segal generalized the notion of movability to arbitrary compact Hausdorff spaces. More recently, Kozlowski and Segal [9] have generalized movability to general topological spaces using a notion of shape which is equivalent to the one developed by Mardesic [12]. In [2] Borsuk introduced the notion of $n$-movability for compact spaces. Kozlowski and Segal generalized this notion to arbitrary compact Hausdorff spaces [8] and then to arbitrary topological spaces [9]. One of the main results of [9] is that an $\mathrm{LC}^{n-1}$ paracompactum is $n$-movable. This is a significant generalization of earlier results about compact metric spaces that are $\mathrm{LC}^{n-1}$ by Borsuk [2], Mardesić [11], and Overton and Segal [15]. The notion of movability and $n$-movability has played an important role in shape theory and the class of movable spaces has been shown to have many useful properties.

It is the purpose of this paper to investigate the properties of the Čech cohomology groups of spaces that are movable and $n$-movable. In an earlier paper [4] the author has studied the properties of the Čech cohomology groups

Received by the editors December 19, 1974.

AMS (MOS) subject classifications (1970). Primary 55D99, 55B05.

Key words and phrases. Shape theory, movable space, $n$-movable space, $\mathrm{LC}^{n-1}$ paracompactum, Čech cohomology.

(1) Supported by NSF grant GP-42664. 
of compact Hausdorff spaces that are movable. In [4] it was shown that if $X$ is a movable compact Hausdorff space, then $\check{H}^{n}(X) /$ Tor $\check{H}^{n}(X)$ is an $\aleph_{1}$-free abelian group for all $n$. In [6] it was shown that for a compact connected abelian topological group $A, A$ is movable if and only if $\check{H}^{1}(A)$ is an $\aleph_{1}$-free abelian group. In [7] these results were used to answer several questions concerning movable compact Hausdorff spaces. In [4] and [5] it was shown that if $X$ is any topological space, which is locally arcwise connected (i.e., $\left.\mathrm{LC}^{0}\right)$, then $\check{H}^{1}(X)$ is an $\aleph_{1}$-free abelian group.

In this paper the results in [4] and [5] are generalized to arbitrary topological spaces. The precise results are stated and proved in $\S \S 3$ and 4 of this paper. The algebraic and topological preliminaries which are needed for the statements and proofs of the main theorems are introduced in $\S \S 1$ and 2. The most important concept introduced is that of an algebraically compact abelian group which we give in §2. The definition of an algebraically compact abelian group would probably not be much help to the reader who is not familiar with the concept. Consequently, in $\$ 2$ we not only define the notion but try to give some idea of the structure of these groups as well. Of course, the reader would do well to consult Fuch's book [3, Chapter VII] where there is an excellent chapter on algebraically compact groups.

As an application of our results we give examples of $n$-movable and movable metric continua which are not shape equivalent to any $\mathrm{LC}^{n-1}$ continuum and in fact not shape equivalent to any $\mathrm{LC}^{n-1}$ paracompactum.

1. Topological preliminaries. In this section we introduce some of the background material that is necessary to state and prove the main results in this paper. Concepts and results from shape theory, algebraic topology, and the theory of abelian groups will be used in this paper. Since many who might be interested in the results of this paper may not have the necessary background in one or more of these areas we will try to give a fairly complete account of what is needed in $\S \S 1$ and 2 . The main theorems and their proofs will then be given in $\S \S 3$ and 4.

Let $T$ denote the category of topological spaces and continuous maps. Let $H$ denote the homotopy category and let $H: T \rightarrow H$ be the homotopy functor. Let $S$ denote the shape category. The objects of $S$ are all topological spaces as in $T$ and $H$. If $X$ and $Y$ are topological spaces, then a shape morphism $F: X \rightarrow Y$ is described in the following manner. Let $p: Y \rightarrow P$ be a continuous map with $P$ any polyhedron. Then $F$ assigns to the homotopy class of $p, H(p)$, a homotopy class of maps $F(H(p))$ from $X$ to $P$. The only assumption made concerning this assignment is that if $p: Y \rightarrow P$ and $q: Y \rightarrow Q$ and $r: Q \rightarrow P$ with $H(p)=$ $H(r) \circ H(q)$ where $P$ and $Q$ are polyhedra, then 


$$
F(H(p))=H(r) \circ F(H(q)) \text {. }
$$

The shape functor $S: T \rightarrow S$ is the identity on the objects of $T$ onto the objects of $S$. If $f: X \rightarrow Y$ is a continuous function, then $S(f) \in \operatorname{Mor}_{S}(X, Y)$ is the function which assigns to each homotopy class $H(p): Y \rightarrow P$ (where $P$ is a polyhedron), the homotopy class of maps

$$
S(f)(H(p))=H(p \circ f): X \rightarrow P .
$$

Clearly $S(f)$ defined in this way is a shape morphism from $X$ to $Y$. Clearly the shape functor factors through the homotopy category.

The shape category $S$ is described by Mardesic in [12]. An equivalent approach due to Kozlowski and Segal is given in [9]. If one's attention is restricted to the category of compact metric spaces, compact Hausdorff spaces, or the category of metric spaces, then the shape category in these cases is the full subcategory of $S$ determined by the objects under consideration. The shape functor for each of these categories is the restriction of $S$ to the appropriate subcategory of $S$.

Let $X$ be a topological space. Then $X$ is said to be movable provided that whenever $p: X \rightarrow P$ is continuous with $P$ a polyhedron, there is a polyhedron $Q$ and maps $q: X \rightarrow Q$ and $s: Q \rightarrow P$ such that

$$
H(p)=H(s) \circ H(q)
$$

and such that whenever $R$ is a polyhedron with $r: X \rightarrow R$ and $t: R \rightarrow Q$ with $H(q)=H(t) \circ H(r)$, then there is a map $u: Q \rightarrow R$ such that

$$
H(r)=H(r \circ t) \circ H(u) \text {. }
$$

This definition of movability agrees with the definition given by Kozlowski and Segal [9] and is equivalent to the one given by Mardesić and Segal [13] for compact Hausdorff spaces.

Let $X$ be a topological space. Then $X$ is said to be $n$-movable provided the following holds: If $p: X \rightarrow P$ is a continuous map with $P$ a polyhedron, then there are a polyhedron $Q$ and continuous maps $q: X \rightarrow Q$ and $s: Q \rightarrow P$ with $H(p)=H(s) \circ H(q)$ such that whenever $R$ is a polyhedron with $r: X \rightarrow R$ and $t: R \rightarrow Q$ continuous maps with

$$
H(t) \circ H(r)=H(q),
$$

then there is a continuous map $u: Q^{(n)} \rightarrow R$ (where $Q^{(n)}$ is the $n$-skeleton of Q) with

$$
H(s \circ t) \circ H(u)=H\left(s \mid Q^{(n)}\right) .
$$

The definition we have given for $n$-movability agrees with Borsuk's notion of $n$ movability for compact metric spaces given in [2] and with the $n$-movability of [8] given for compact Hausdorff spaces. In [9] it is shown that movability and $n$-movability are shape invariants. 
A space $X$ is said to be locally $k$-connected provided that for each $\boldsymbol{x} \in X$ and neighborhood $U$ of $x$, there is a neighborhood $V$ of $x$ contained in $U$ such that every map $f: S^{k} \rightarrow V$ extends to a map $F: B^{k+1} \rightarrow U$ where $S^{k}=\partial B^{k+1}$ is the $k$-sphere and $B^{k+1}$ is the $(k+1)$-ball. A space is said to be LC $^{n}$ provided it is locally $k$-connected for all $0 \leqslant k \leqslant n$. In [9] it is shown that any $\mathrm{LC}^{n-1}$ paracompactum is $n$-movable. This was proved for $\mathrm{LC}^{n-1}$ compact metric spaces by Borsuk in [2].

As in [9] we assume all paracompact spaces to be Hausdorff.

2. Algebraic preliminaries. Let $X$ be a topological space. We let $H^{n}(X)$ and $H_{n}(X)$ denote $n$-dimensional singular cohomology and homology of $X$ with integer coefficients. As noted earlier we let $\check{H}^{n}(X)$ and $\check{H}_{n}(X)$ denote $n$-dimensional Čch cohomology and homology with integer coefficients and based on the numerable covers of $X$. One of the principal tools in this paper is the Universal Coefficient Theorem for singular cohomology. See [16, Theorem 3, p. 243] for a reference.

\subsection{THEOREM. There is a functorial short exact sequence}

$$
0 \rightarrow \operatorname{Ext}\left(H_{q-1}(X), Z\right) \rightarrow H^{q}(X) \rightarrow \operatorname{Hom}\left(H_{q}(X), Z\right) \rightarrow 0
$$

and this sequence is split.

It is well known that this theorem is not true for Čech cohomology. This will be clear from results and examples in this paper also. The Universal Coefficient Theorem for singular cohomology not only shows that $H^{q}(X)$ is determined by $H_{q-1}(X)$ and $H_{q}(X)$, but also indicates that $H^{q}(X)$ must have a certain algebraic structure which we now proceed to point out.

2.2. Definition. Let $H$ be an abelian group. Then $H$ is said to be algebraically compact provided there is an abelian group $G$ such that $H \oplus G$ is isomorphic to a compact Hausdorff topological group.

The algebraically compact abelian groups turn out to be precisely the class of pure-injective abelian groups. They are also equivalent to the class of abelian groups which are direct summands of direct products of cocyclic groups [3, Theorem 38.1, p. 160]. The basic background concerning algebraically compact abelian groups will be found in Chapter VII of Fuchs [3]. We will give most of the background results in this section that will be needed in the later sections of the paper.

It is known that every divisible group is algebraically compact. Thus every algebraically compact abelian group $H$ is isomorphic to $D \oplus G$ where $D$ is the divisible subgroup of $H$ and $G$ is a reduced algebraically compact group. Furthermore, if $H$ is any abelian group, then $H$ is algebraically compact if and only if 
its reduced part is a reduced algebraically compact group. This reduces the study of the structure of algebraically compact groups to the study of reduced algebraically compact groups since the structure of divisible groups is well known.

What makes algebraically compact groups significant in this study is the following result $[3, \mathrm{~N}, \mathrm{p} .224]$.

2.3. THEOREM. If $H$ is a torsion free abelian group and $C$ is any abelian group, then $\operatorname{Ext}(C, H)$ is algebraically compact.

We now analyze the structure of $\operatorname{Hom}\left(H_{q}(X), Z\right)$ in the Universal Coefficient Theorem. First we define the significant concept.

2.4. Definition. Let $H$ be an abelian group. Then $H$ is $\aleph_{1}$-free if every subgroup $G$ of $H$ whose cardinality is countable is free abelian.

A famous theorem of Pontryagin states that a torsion free abelian group $H$ is $\aleph_{1}$-free if and only if every subgroup of finite rank is free [3, Theorem 19.1, p. 93]. This implies that a torsion free abelian group $H$ is $\aleph_{1}$-free if and only if $H$ has property $L$. (See [4] or [6] for the definition of property $L$. The notion of property $L$ is due to Pontryagin.)

Clearly any subgroup of a group which is $\aleph_{1}$-free is also $\aleph_{1}$-free. However, for any abelian group $H, \operatorname{Hom}(H, Z)$ is a subgroup of a direct product of the integers $\Pi_{h \in H} Z$. By [3, Theorem 19.2, p. 94] any direct product of the integers is $\aleph_{1}$-free. Thus we have the following theorem which we state for reference.

\subsection{TheOREM. For any abelian group $H, \operatorname{Hom}(H, Z)$ is $\aleph_{1}$-free.}

Theorems 2.3 and 2.5 tell us quite a bit about the algebraic structure of any group which is a singular cohomology group $H=H^{q}(X)$ for some space $X$. If one first factors out the divisible subgroup $D$ of $H$, then $D$ must be a subgroup of $\operatorname{Ext}\left(H_{q-1}(X), Z\right)$. This is because $\operatorname{Hom}\left(H_{q}(X), Z\right)$ in the Universal Coefficient Theorem is $\aleph_{1}$-free and contains only the trivial divisible subgroup. Thus we have

$$
\operatorname{Ext}\left(H_{q-1}(X), Z\right) \simeq D \oplus G
$$

where $G$ is some reduced algebraically compact subgroup of $H$. Thus $H$ is isomorphic to $D \oplus G \oplus K$ where $D$ is a divisible group, $G$ is a reduced algebraically compact group and $K$ is an $\aleph_{1}$-free group.

We will now show that every algebraically compact subgroup $A$ of $H$ is contained in $D \oplus G=\operatorname{Ext}\left(H_{q-1}(X), Z\right)$ and thus the subgroup $\operatorname{Ext}\left(H_{q-1}(X), Z\right)$ can be characterized algebraically as the largest algebraically compact subgroup of $H$. To show this we need some more results about reduced algebraically compact groups.

For any abelian group $G$, the first Ulm subgroup of $G$ is $G^{1}=\bigcap_{n-1}^{\infty} n G$. The first result we need is [3, Corollary 39.2, p. 163]. 
2.6. THEOREM. If $H$ is a reduced algebraically compact abelian group and if $G$ is a subgroup of $H$ such that $(H / G)^{1}=0$, then both $G$ and $H / G$ are reduced algebraically compact groups.

Now if $G \subset H$, then clearly $G^{1} \subset H^{1}$. Couple this with the fact that a reduced algebraically compact group $H$ has $H^{1}=0$ and we have the following corollary.

2.7. COROLLARY. If $h: H \rightarrow G$ is a homomorphism between reduced algebraically compact abelian groups, then $h(H)$ is a reduced algebraically compact group as well.

If an abelian group $H$ is torsion free, then its first Ulm subgroup $H^{1}$ is just the maximal divisible subgroup of $H$. If $H$ is $\aleph_{1}-$ free, then $H^{1}$ is clearly 0 . Thus if $h: H \rightarrow G$ is a homomorphism between abelian groups with $H$ reduced algebraically compact and $G \aleph_{1}$-free, then $h(H)^{1}=0$ and thus $h(H)$ is a reduced algebraically compact group. However, the next result implies that $h(H)$ is in fact just $0, h(H)=0$.

2.8. THEOREM [3, COROLlARY 40.4, p. 169]. Every reduced algebraically compact group $\neq 0$ contains a direct summand isomorphic to $J_{p}$ or $Z_{p}$ for some prime $p$ and some positive integer $k$ where $J_{p}$ is the p-adic integers.

Theorem 2.8 implies that $h(H)=0$ in the above discussion since $h(H)$ must be torsion free as a subgroup of $G$. We summarize.

2.9. COROLlARY. If $h: H \rightarrow G$ is a homomorphism between abelian groups with $H$ reduced algebraically compact and $G \aleph_{1}$-free, then $h=0$.

2.10. Corollary. If $h: H \rightarrow G$ is a homomorphism between abelian groups with $H$ algebraically compact and $G \aleph_{1}$-free, then $h=0$.

Proof. Let $H=D \oplus A$ where $D$ is the maximal divisible subgroup of $H$ and $A$ is a reduced algebraically compact group. Now $h(A)=0$ by Corollary 2.9. On the other hand $h(D)$ is a divisible subgroup of $G$ and since $G^{1}=0, h(D)=0$ also. Thus $h=0$.

These results combine to give us the following additional theorem about the algebraic structure of a group which is a singular cohomology group of some space.

2.11. THEOREM. Let $H$ be any group which is a singular cohomology group $H^{q}(X)$ of a space $X$. Then $H$ contains an algebraically compact subgroup $E_{H}$ which contains all other algebraically compact subgroups of $H$. Furthermore, $H / E_{H}$ is $\aleph_{1}$-free.

Proof. If $H=H^{q}(X)$, then $E_{H}$ corresponds to $\operatorname{Ext}\left(H_{q-1}(X), Z\right)$ in 
$H^{q}(X)$. By the Universal Coefficient Theorem $H / E_{H}$ is $\aleph_{1}$-free. All that remains to be shown is that $E_{H}$ contains all the algebraically compact subgroups of $H$. Let $A$ be an algebraically compact subgroup of $H$. The image of $A$ in $H / E_{H}$ must be zero by Corollary 2.10. Thus $A$ is contained in $E_{H}$.

Theorem 2.11 tells us that if we are given a group $H$ and told that it is a singular cohomology group $H^{q}(X)$ for some space $X$, then we can reconstruct the short exact sequence given in the Universal Coefficient Theorem without knowing the groups $H_{q-1}(X)$ or $H_{q}(X)$.

We quote one last theorem concerning reduced algebraically compact groups which we will need in $\S 4$. An abelian group $G$ which is pure torsion is bounded if there is an integer $n$ such that $n x=0$ for all $x \in G$.

2.12. THEOREM [3, COROLLARY 40.3, p. 169]. If a reduced algebraically compact group is torsion, then it is bounded.

3. Movable and $n$-movable spaces. Our main theorems in this section are the following two theorems.

3.1. THEOREM. Let $X$ be a movable space. Then for each $n, \stackrel{\Upsilon}{H}^{n}(X)$ has a subgroup $E$ which is the union of all algebraically compact subgroups of $\stackrel{H}{H}^{n}(X)$ and $E$ has the property that $\mathscr{H}^{n}(X) / E$ is an $\aleph_{1}$-free group.

3.2. THEOREM. Let $X$ be an $n$-movable space. Then for each $0 \leqslant k \leqslant n$, $\stackrel{\Upsilon}{H}^{k}(X)$ has a subgroup $E$ which is the union of all the algebraically compact subgroups of $\check{H}^{k}(X)$ and $E$ has the property that $\check{H}^{k}(X) / E$ is an $\aleph_{1}$-free group.

In case $X$ is compact, these theorems have the following corollaries.

3.3. Corollary. Let $X$ be a movable compact Hausdorff space. Then for each $n, \check{H}^{n}(X) /$ Tor $\check{H}^{n}(X)$ is an $\aleph_{1}$-free group.

3.4. Corollary. Let $X$ be an n-movable compact Hausdorff space. Then for each $0 \leqslant k \leqslant n, \check{H}^{k}(X) /$ Tor $\check{H}^{k}(X)$ is an $\aleph_{1}$-free group.

Corollary 3.3 was first proved in [4]. Before proving these theorems and their corollaries we will need to introduce some notation and state a result from [9]. After we have stated and proved the above theorems and corollaries we will include some additional related results in this section as well.

Let $X$ be a topological space. If $U$ is an open cover of $X$, then we let $N(U)$ denote the nerve of the cover. We will also let $N(U)$ denote the geometric realization of this nerve. Context will make it clear which of these objects is being referred to. If $V$ is an open cover which refines $U$, we let $\pi_{u V}: N(V) \rightarrow$ $N(U)$ denote the projection map. Of course, the projection map is unique up to homotopy. For any open cover $U$ of $X$, a continuous map $f: X \rightarrow N(U)$ is 
called a barycentric map provided $f^{-1}\left(\operatorname{St}\left(v_{U}\right)\right) \subset U$ for all $U \in U$ where $v_{U}$ is the vertex in $N(U)$ associated with $U$. A cover $U$ is said to be numerable if there is a partition of unity subordinate to it. It is well known that an open cover $U$ admits a barycentric map $f: X \rightarrow N(U)$ if and only if it is a numerable cover. We now state a result which is essentially in [9].

3.5. THEOREM. A topological space is movable if and only if for any numerable cover $U$ of $X$, there is a numerable open cover $V$ refining $U$ such that for any numerable open cover $W$ refining $V$, there is a map $\lambda=\lambda^{v \omega}: N(V) \rightarrow$ $N(\omega)$ such that $\pi_{u \omega} \circ \lambda$ is homotopic to $\pi_{u v}$. A topological space is n-movable if and only if for every numerable open cover $U$ of $X$, there is a numerable open cover $U$ refining $U$ such that for any numerable open cover $W$ refining $U$ there is a map $\lambda: N(V)^{(n)} \rightarrow N(W)$ such that $\pi_{u \omega} \circ \lambda$ is homotopic to $\pi_{u V} \mid N(V)^{(n)}$.

This is essentially Theorem 4.3 of [9]. In Theorem 4.3 of [9] the space $X$ was assumed paracompact and the covers $U, V$, and $W$ were simply arbitrary open covers. However, in examining the proof of Theorem 4.3 of [9] one can see that the assumption that $X$ was paracompact was used only in guaranteeing that the covers $U, V$, and $W$ were numerable. With this in mind the reader can easily verify that the proof of Theorem 4.3 of [9] actually proves Theorem 3.5 above.

We are now ready to prove the main theorems and corollaries of this section.

Proof of Theorem 3.1. Let $X$ be a topological space and let $\operatorname{Cov}(X)$ denote the collection of numerable covers of $X$. Let $N(X)$ denote the inverse system of nerves of numerable covers and homotopy classes of projection maps $N(X)=\left\{N(U) ; H\left(\pi_{U V}\right) ; U<U \in \operatorname{Cov}(X)\right\}$. The $n$-dimensional Čech cohomology based on numerable covers is the direct limit of the system of groups and homomorphisms

$$
\left\{H^{n}(N(U)) ; \pi_{u v}^{*} ; U<U \in \operatorname{Cov}(X)\right\}
$$

Because of the functoriality of the Universal Coefficient Theorem for singular cohomology, there is associated with $N(X)$ a direct system of short exact sequences of abelian groups

$$
\left\{0 \rightarrow \operatorname{Ext}\left(H_{n-1}(N(U)), Z\right) \rightarrow H^{n}(N(U)) \rightarrow \operatorname{Hom}\left(H_{n}(N(U)), Z\right) \rightarrow 0\right\} .
$$

Now the direct limit of a direct system of exact sequences of abelian groups is also an exact sequence. Thus if we let $E$ be the direct limit of $\left\{\operatorname{Ext}\left(H_{n-1}(N(U)), Z\right)\right\}$ and $H$ denote the direct limit of $\left\{\operatorname{Hom}\left(H_{n}(N(U)), Z\right)\right\}$, then we have a short exact sequence

$$
0 \rightarrow E \rightarrow \check{H}^{n}(X) \rightarrow H \rightarrow 0 .
$$

Now suppose that $X$ is a movable space. Then we will show that $(*) E$ is a union of 
algebraically compact groups and $H$ is an $\aleph_{1}$-free abelian group. Assume (*) for a moment and we will show that Theorem 3.1 follows from this. Identify $E$ with its image in $\check{H}^{n}(X)$. Then this will be the $E$ in Theorem 3.1. Clearly $\check{H}^{n}(X) / E$ is $\aleph_{1}$-free since it is isomorphic to $H$ which by (*) is $\aleph_{1}$-free. By (*) $E$ is a union of algebraically compact groups. So, all that remains to be shown is that if $A \subset \check{H}^{n}(X)$ is an algebraically compact group, then $A \subset E$. However, the image of $A$ in $\check{H}^{n}(X) / E$ is zero by Corollary 2.10. Thus $A \subset E$. Thus Theorem 3.1 will follow once we have shown (*). We now prove (*) by a sequence of claims.

Claim 1. The group $E$ is a union of algebraically compact abelian groups.

Proof of Claim 1. The group $E$ is the direct limit of the direct system $\left\{\operatorname{Ext}\left(H_{n-1}(N(U)), Z\right)\right\}$. To show that $E$ is a union of algebraically compact groups let us analyze the direct system more closely. Let $E_{u}=\operatorname{Ext}\left(H_{n-1}(N(U)), Z\right)$. Let $\gamma^{u v}: E_{u} \rightarrow E_{v}$ be the induced homomorphism when $V<U \in \operatorname{Cov}(X)$. Let $D_{U}$ denote the maximum divisible subgroup of $E_{u}$. Then for $V<U \in \operatorname{Cov}(X)$, $\gamma^{u v}\left(D_{u}\right) \subset D_{v}$. Let $A_{u}$ denote the group $E_{u} / D_{u}$. Then the homomorphisms $\gamma^{u} v_{\text {induce homomorphisms of short exact sequences. }}$

$$
\begin{aligned}
0 \rightarrow D_{u} & \rightarrow E_{u} \rightarrow A_{u} \rightarrow 0 \\
& \downarrow \\
0 & \downarrow D_{v} \rightarrow E_{v} \rightarrow A_{v} \rightarrow 0
\end{aligned}
$$

If we let $D$ denote the direct limit of the direct system $\left\{D_{U}\right\}$ and $A$ denote the direct limit of the direct system $\left\{A_{u}\right\}$, then we have the following exact sequence:

$$
0 \rightarrow D \rightarrow E \rightarrow A \rightarrow 0 \text {. }
$$

Clearly $D$ is a divisible group, so the sequence splits and $E \simeq D \oplus A$. We will now use the movability of $X$ to show that $A$ is the union of reduced algebraically compact groups. Then if $A=\bigcup_{\gamma \in \Gamma} A_{\gamma}$ with each $A_{\gamma}$ an algebraically compact group, then $E=\bigcup_{\gamma \in \Gamma} D \oplus A_{\gamma}$. But $D \oplus A_{\gamma}$ is the direct sum of two algebraically compact groups and hence algebraically compact. So Claim 1 will follow from Claim 2.

Claim 2. The group $A$ is a union of reduced algebraically compact groups.

Proof of Claim 2. Let $V<U \in \operatorname{Cov}(X)$ and let $\bar{\gamma} u v: A_{U} \rightarrow A_{V}$ be the induced homomorphism. Now let $U$ be a numerable open cover of $X$ and let $V$ be a numerable open cover of $X$ satisfying the conditions of Theorem 3.5 by the movability of $X$. We now claim that for any numerable open cover $W$ refining $V, \bar{\gamma}^{v \omega} \mid \bar{\gamma}^{u v}\left(A_{u}\right)$ is one-to-one. We will prove this as Claim 3. Claim 3 will will then imply that if we let $\left\{\bar{\gamma}^{u}: A_{u} \rightarrow A\right\}$ denote the injection maps making $A$ the direct limit of the direct system $\left\{A_{U}\right\}$, then $\bar{\gamma}^{U}(A)$ will be isomorphic to $\bar{\gamma} u v\left(A_{u}\right)$. However, according to Corollary $2.7, \bar{\gamma} u v\left(A_{u}\right)$ is a reduced algebraically 
compact group. Thus $\bar{\gamma}^{u}\left(A_{U}\right)$ will be a reduced algebraically compact group as well. Since $A=\bigcup_{u \in \operatorname{Cov}(X)} \bar{\gamma}^{u}\left(A_{u}\right)$ this will prove Claim 2. So Claim 2 will follow from Claim 3.

ClaIM 3. The homomorphism $\bar{\gamma} v w \mid \bar{\gamma} U v\left(A_{U}\right): \bar{\gamma} v v\left(A_{U}\right) \rightarrow A$ is one-to-one.

Proof of Claim 3. Let $a \in \bar{\gamma}^{U v}\left(A_{U}\right)$ and let $b \in A_{U}$ with $\bar{\gamma} u v(b)=a$. Let $W$ be any numerable open cover refining $V$. Suppose that $\bar{\gamma} v w(a)=0$. By the choice of $V$, there is a continuous map $\lambda: N(V) \rightarrow N(W)$ so that

$$
H\left(\pi_{u v}\right)=H\left(\pi_{u w}\right) \circ H(\lambda) \text {. }
$$

Now $\lambda$ induces a homomorphism $\bar{\gamma}: A_{\omega} \rightarrow A_{v}$ so that

$$
\bar{\gamma} \circ \bar{\gamma} u w=\bar{\gamma} u v \text {. }
$$

This implies that $a=\bar{\gamma}^{u v(b)}=\bar{\gamma} \circ \bar{\gamma}^{u \omega}(b)=\bar{\gamma} \circ \bar{\gamma}^{v \omega} \circ \bar{\gamma}^{u v}(b)=\bar{\gamma} \circ \bar{\gamma}^{v \omega}(a)=$ $\bar{\gamma}(0)=0$. That is, for any numerable open cover $W$ refining $V$, the kernel of $\bar{\gamma} v u\left(\bar{\gamma} U V\left(A_{u}\right)\right.$ is zero and thus Claim 3 is proved. We have now completely proved Claim 2 and Claim 1.

To complete the proof of Theorem 3.1 we need to prove the latter part of the statement (*). This we will prove as Claim 4.

Claim 4. The group $H$ is $\aleph_{1}$-free.

Proof of Claim 4. To prove Claim 4 we first note that since $H$ is the direct limit of torsion free groups, $H$ must be torsion free. This allows us to apply Pontryagin's criterion of $\aleph_{1}$-freeness [3, Theorem 19.1, p. 93] to say that $H$ is $\aleph_{1}$-free if and only if every subgroup of $H$ of finite rank is free. So let $G \subset H$ be a subgroup of finite rank. Then Claim 4 will follow from Claim 5 .

Claim 5. The group $G$ is free.

Proof of Claim 5. Since the group $G$ is of finite rank, it is countable. Thus if $G$ is $\aleph_{1}$-free, then it is free. We will show that $G$ is free by showing that $G$ is a subgroup of an $\aleph_{1}$-free subgroup of $H$. To show this let $\left\{g_{1}, \ldots, g_{m}\right\}$ be a maximal linearly independent set of elements in $G$. Let

$$
H_{u}=\operatorname{Hom}\left(H_{n}(N(U)), Z\right)
$$

for each $U \in \operatorname{Cov}(X)$ and let $h^{u v}: H_{u} \rightarrow H_{u}$ denote the homomorphisms induced by the projection maps $\pi_{u v}$. Let $h^{u}: H_{u} \rightarrow H$ denote the injection homomorphisms making $H$ the direct limit of the direct system $\left\{H_{U}\right\}$. Now let $U \in$ $\operatorname{Cov}(X)$ be such that $h^{u}(H) \supset\left\{g_{1}, \ldots, g_{m}\right\}$. Then let $V$ be a numerable open cover refining $U$ given by Theorem 3.5 by the movability of $X$. Then Claim 5 will follow from the following two claims. First of all, $h^{V}\left(H_{v}\right)$ contains the group $G$. Secondly, the group $h^{\nu}\left(H_{V}\right)$ is $\aleph_{1}$-free. These will be proved as Claim 6 and Claim 7.

Claim 6. The group $G$ is contained in $h^{V}\left(H_{V}\right)$.

Proof of Claim 6. Every element of $G$ is a root of some linear combina- 
tion with integer coefficients of the elements $g_{1}, \ldots, g_{m}$. Thus it will be sufficient to show that $h^{v}\left(H_{v}\right)$ contains all the roots of $h^{u}\left(H_{u}\right)$ in $H$. That is, if $k g \in h^{u}\left(H_{u}\right)$ for some positive integer $k$ and $g \in H$, then we need to show that $g \in h^{v}\left(H_{V}\right)$. So, suppose that $k g \in h^{u}\left(H_{u}\right)$. Then $k g=h^{u}(a)$ for some $a \in H_{u}$. Now let $W \in \operatorname{Cov}(X)$ refine $V$ such that, for some $b \in H$, $h^{W}(b)=g$. Now $h^{w}(k b)=k g=h^{u}(a)$. Thus there is a $y \in \operatorname{Cov}(X)$ such that $y$ refines $W$ and $h^{w y}(k b)=h^{u y}(a)$. Now let $\lambda: N(y) \rightarrow N(V)$ be the continuous map given in Theorem 3.5 such that $\pi_{u v}$ is homotopic to $\pi_{u y} \circ \lambda$. Let $h$ : $H_{y} \rightarrow H_{v}$ denote the homomorphism induced by $\lambda$. Then let $c \in H_{v}$ be defined by $c=h \circ h^{\text {Wy }}(b)$. Then $k c=k h \circ h^{\omega y}(b)=h \circ h^{\omega y}(k b)=h \circ h^{\text {uy }}(a)=h^{u v}(a)$. This implies that $k h^{V}(c)=h^{U}(a)=k g$. That is, $h^{V}(c)$ is a $k$ th root of $k g$. But since $H$ is torsion free, $k$ th roots are unique. Therefore $h^{V}(c)=g$ and $g \in h^{V}\left(H_{v}\right)$. Therefore $G \subset h^{V}\left(H_{v}\right)$ and Claim 6 is proved.

Claim 7. The group $h^{V}\left(H_{v}\right) \subset H$ is $\aleph_{1}$-free.

Proof of Claim 7. Let $W \in \operatorname{Cov}(X)$ be the refinement of $V$ satisfying the conditions of Theorem 3.5 by the movability of $X$ (considering $V$ as the $U$ in that theorem and $W$ satisfying the conditions that $V$ does in Theorem 3.5). Then as in the Proof of Claim 3, for any $y$ refining $W$, the homomorphism $h^{w y} \mid h^{v \omega}\left(H_{v}\right)$ is one-to-one. Thus $h^{w} \mid h^{v \omega}\left(H_{v}\right)$ is also one-to-one. Therefore $h^{V}\left(H_{v}\right)$ is isomorphic to a subgroup of $H_{w}$. Since $H_{w}$ is $\aleph_{1}$-free, this implies that $h^{V}\left(H_{v}\right)$ is also $\aleph_{1}$-free and Claim 7 is proved. Claim 6 and Claim 7 complete the proof of Claim 5. The Proof of Theorem 3.1 is complete.

We now proceed to the Proof of Theorem 3.2.

Proof of Theorem 3.2. We need to consider two cases in the proof. First we indicate the proof for $\check{H}^{k}(X)$ for $k \leqslant n-1$. A separate argument is needed for the case that $k=\dot{n}$.

Case I. $k \leqslant n-1$. This case follows from the Proof of Theorem 3.1. To adapt the Proof of Theorem 3.1 to this case one needs to observe that for every $U \in \operatorname{Cov}(X), H_{k}\left(N(U)^{(n)}\right) \simeq H_{k}(N(U)), H_{k-1}\left(N(U)^{(n)}\right) \simeq H_{k-1}(N(U))$, and $H^{k}(N(U)) \simeq H^{k}\left(N(U)^{(n)}\right)$ with the isomorphisms induced by the inclusion map $N(U)^{(n)} \subset N(U)$. Then one uses the $\lambda$ 's given in Theorem 3.5 given by $X n$-movable to induce the required homomorphisms rather than the $\lambda$ 's given by Theorem 3.5 for $X$ movable in the Proof of Theorem 3.1. We leave it to the reader to carry out the details.

Case II. $k=n$. In this case a little more must be said although some parts of the Proof of Theorem 3.1 can be adapted to shorten the proof in this case as well. As in the Proof of Theorem 3.1 we consider the direct system of short exact sequences of abelian groups:

$$
\left\{0 \rightarrow \operatorname{Ext}\left(H_{n-1}(N(U)), Z\right) \rightarrow H^{n}(N(U)) \rightarrow \operatorname{Hom}\left(H_{n}(N(U)), Z\right) \rightarrow 0\right\}
$$


Let $E$ be the direct limit of the direct system $\left\{\operatorname{Ext}\left(H_{n-1}(N(U)), Z\right)\right\}$ and $H$ be the direct limit of the direct system $\left\{\operatorname{Hom}\left(H_{n}(N(U)), Z\right)\right\}$ as in the Proof of Theorem 3.1. Then we have an exact sequence

$$
0 \rightarrow E \rightarrow \stackrel{\check{H}}{n}^{n}(X) \rightarrow H \rightarrow 0 .
$$

As in the Proof of Theorem 3.1 we will show that $E$ is a union of algebraically compact groups and that $H$ is $\aleph_{1}$-free. Once this is shown it will follow that every algebraically compact subgroup of $H^{n}(X)$ will be contained in $E$ and the proof will be complete in this case as well.

ClaIm 1. The group $E$ is a union of algebraically compact subgroups.

Proof of Claim 1. This follows by adapting the Proof of Theorem 3.1 as indicated in Case I. We leave it to the reader to carry out the details.

Claim 2. The group $H$ is $\aleph_{1}$-free.

Proof of Claim 2. As in the Proof of Theorem 3.1 we show that $H$ is $\aleph_{1}$-free by showing that each subgroup of finite rank is free. However, our method of proof of this fact in this case is different from the one in the proof of Theorem 3.1.

The Proof of Claim 2 will be by contradiction. Suppose that $H$ is not $\aleph_{1}$ free. Let $G$ be a subgroup of $H$ which has finite rank $m$ and which is not free. Let $\left\{g_{1}, \ldots, g_{m}\right\} \subset G$ be a maximal linearly independent set of elements in $G$. Let $H_{u}=\operatorname{Hom}\left(H_{n}(N(U)), Z\right)$ for each $U \in \operatorname{Cov}(X)$ and let $\left\{h^{u}: H_{U} \rightarrow H\right\}$ be the collection of homomorphisms making $H$ the direct limit of $\left\{H_{U}\right\}$. Denote the bonding homomorphisms by $h^{u V}: H_{U} \rightarrow H_{V}$ for $U<U \in \operatorname{Cov}(X)$. Now let $u \in \operatorname{Cov}(X)$ so that $\left\{g_{1}, \ldots, g_{m}\right\} \subset h^{U}\left(H_{u}\right)$. Then let $V \in \operatorname{Cov}(X)$ be a refinement of $U$ which satisfies the conditions of Theorem 3.5 by the $n$-movability of $X$. Let $h_{i} \in H$ be such that $h^{\mathrm{U}}\left(h_{i}\right)=g_{i}$. Then $\left\{h_{1}, \ldots, h_{m}\right\}$ is a linearly independent set of elements in $H_{U}$. Note that the homomorphism $H_{n}\left(N(V)^{(n)}\right.$ ) $\rightarrow H_{n}(N(V))$ induced by the inclusion $N(V)^{(n)} \subset N(V)$ is onto. Thus the induced homomorphism

$$
H_{V} \stackrel{e}{\rightarrow} \operatorname{Hom}\left(H_{n}\left(N(V)^{(n)}\right), Z\right)
$$

must be one-to-one. Now let $f_{i}=e\left(h^{U}\left(h_{i}\right)\right)$ for $i=1, \ldots, m$. Then $\left\{f_{1}, \ldots, f_{m}\right\}$ is a linearly independent set of elements in $\operatorname{Hom}\left(H_{n}\left(N(V)^{(n)}\right), Z\right)$. Now let $W \in$ $\operatorname{Cov}(X)$ refine $V$ and let $\left.p_{\omega}: H_{\omega} \rightarrow \operatorname{Hom}\left(N(V)^{(n)}\right), Z\right)$ be the homomorphism induced by the $\lambda$ given by the $n$-movability of $X$ in Theorem 3.5. Then, for each such $\omega, p_{\omega} \circ h^{U \omega}=e \circ h^{U v}$. Now we are ready to show the contradiction in assuming that $H$ is not $\aleph_{1}$-free. Since $\left\{g_{1}, \ldots, g_{m}\right\}$ is linearly independent in $G \subset H$, one can define a unique homomorphism $p$ from the subgroup of $G$ generated by $\left\{g_{1}, \ldots, g_{m}\right\}$ into $\operatorname{Hom}\left(H_{n}\left(N(V)^{(n)}\right), Z\right)$ by $p\left(g_{i}\right)=f_{i}$. Again, using the linear independence of $\left\{g_{1}, \ldots, g_{m}\right\}$ one can extend the homomorphism $p$ to a maximal subgroup $G_{p}$ of $G$. We call the extended homomorphism $p$ also. 
Since $\left\{f_{1}, \ldots, f_{m}\right\}$ is linearly independent, the image of $G_{p}$ in

$$
\operatorname{Hom}\left(H_{n}\left(N(V)^{(n)}\right), Z\right)
$$

will be isomorphic to $G_{p}$. Therefore $G_{p}$ is isomorphic to a subgroup of Hom $\left(H_{n}\left(N(V)^{(n)}\right), Z\right)$ of finite rank and thus $G_{p}$ is a free abelian group since $\operatorname{Hom}\left(H_{n}\left(N(V)^{(n)}\right), Z\right)$ is $\aleph_{1}$-free. We will obtain our contradiction by showing that $G_{p}=G$.

CLAIM 3. The group $G_{p}$ is all of $G$.

Proof of Claim 3. Let $g \in G$ and with $g \notin G_{p}$. Then for some positive integer $k, k g$ is in the group generated by $\left\{g_{1}, \ldots, g_{m}\right\}$. Furthermore, $p(k g)$ cannot have a $k$ th root $a$ in $\operatorname{Hom}\left(H_{n}\left(N(V)^{(n)}\right), Z\right)$ or the homomorphism $p$ could be extended to the group generated by $G_{p} \cup\{g\}$ by sending $g$ to $a$. This would contradict the maximality of $G_{p}$. Let $W \in \operatorname{Cov}(X)$ be a refinement of $U$ such that $g \in h^{\omega}\left(H_{\omega}\right)$ and let $h^{\omega}(b)=g$. Then $h^{\omega}(k b)=k g$ and thus $h^{\omega}(k b)=h^{u}(c)$ for some $c$ in the group generated by $\left\{h_{1}, \ldots, h_{m}\right\}$ in $H_{u}$. This implies that there is a $y \in \operatorname{Cov}(X)$ refining $W$ such that $h^{W y}(k b)=h^{W y}(c)$. Then $p_{y}\left(h^{W y}(b)\right)$ is a $k$ th root of $e^{u V}(c)$ in $\operatorname{Hom}\left(H_{n}\left(N(V)^{(n)}\right), Z\right)$. This is because $k p_{y}\left(h^{(W y}(b)\right)=$ $p_{y}\left(h^{W y}(k b)\right)=p_{y}\left(h^{u y}(c)\right)=e h^{u V}(c)$. This contradicts the fact that $p(k g)=$ eh ${ }^{W W}(c)$ did not contain a $k$ th root in $\operatorname{Hom}\left(H_{n}\left(N(V)^{(n)}\right), Z\right)$. This contradiction shows that $G_{p}=G$. Claim 3 shows that $G$ is a free group, which is a contradiction. This contradiction shows that $H$ must be $\aleph_{1}$-free. The proof that $H$ is $\aleph_{1}$-free completes the Proof of Theorem 3.2 in Case II and we are finished with the Proof of Theorem 3.2.

We will now indicate the proofs of Corollaries 3.3 and 3.4.

Proof of Corollary 3.3. In the Proof of Theorem 3.1, one need not use the complete collection of numerable covers of $X, \operatorname{Cov}(X)$, but only a cofinal collection of those covers. If $X$ is compact, then one simply uses $\operatorname{Cov}^{f}(X)$, the finite numerable covers of $X$ and observes that $\operatorname{Cov}^{f}(X)$ is cofinal in $\operatorname{Cov}(X)$ for $X$ compact and thus $\mathscr{H}^{n}(X)$ is the direct limit of $\left\{H^{n}(N(U)) ; \pi_{u v}^{*} ; V<U \in\right.$ $\operatorname{Cov}^{f}(X)$ \}. However, in this case $N(U)$ is a finite polyhedron for all $U \in \operatorname{Cov}^{f}(X)$. Thus when one considers the direct system of short exact sequences

$$
\left\{0 \rightarrow \operatorname{Ext}\left(H_{n-1}(N(U)), Z\right) \rightarrow H^{n}(N(U)) \rightarrow \operatorname{Hom}\left(H_{n}(N(U)), Z\right) \rightarrow 0\right\}
$$

with $U \in \operatorname{Cov}^{f}(X)$, then all the groups are finitely generated and $\operatorname{Ext}\left(H_{n-1}(N(U)), Z\right)$ corresponds to the torsion part of $H^{n}(N(U))$ and $\operatorname{Hom}\left(H_{n}(N(U)), Z\right)$ corresponds to $H^{n}(N(U)) /$ Tor $H^{n}(N(U))$. Consequently, in the Proof of Theorem 3.1, the direct limit exact sequence

corresponds to

$$
0 \rightarrow E \rightarrow \check{r}^{n}(X) \rightarrow H \rightarrow 0
$$

$$
0 \rightarrow \operatorname{Tor} \check{H}^{n}(X) \rightarrow \check{H}^{n}(X) \rightarrow \check{H}^{n}(X) / \operatorname{Tor} \check{H}^{n}(X) \rightarrow 0 .
$$


Thus for $X$ compact and movable, the group $E$ in Theorem 3.1 is not only a union of algebraically compact groups, but in fact a union of finite groups and is just the torsion part of $\check{H}^{n}(X)$. The group $H$ will be $\aleph_{1}$-free for $X$ movable and consequently we have shown that $\check{H}^{n}(X) /$ Tor $\check{H}^{n}(X)$ is $\aleph_{1}$-free. This proves Corollary 3.3.

The Proof of Corollary 3.4 proceeds in a similar fashion to the Proof of Corollary 3.3. We now state some further corollaries.

3.6. Corollary. Let $X$ be a movable compact Hausdorff space. Then if $\check{H}^{n}(X)$ is torsion free for some $n$, then $\check{H}^{n}(X)$ is $\aleph_{1}$-free for that $n$.

3.7. Corollary. Let $X$ be an n-movable compact Hausdorff space. Then if $0 \leqslant k \leqslant n$ and $\check{H}^{k}(X)$ is torsion free, then $\check{H}^{k}(X)$ is an $\aleph_{1}$-free group for that $k$.

3.8. COROLlary. Let $X$ be an arbitrary movable space. Then if $\check{H}^{n}(X)$ is torsion free and does not contain a copy of the p-adic integers $J_{p}$ for some prime $p$, then $\check{H}^{n}(X)$ has the form $D \oplus A$ where $D$ is a vector space over the rationals and $A$ is $\aleph_{1}$-free.

Proof. From Theorem 3.1 we can assume that $\check{H}^{n}(X)$ contains a subgroup $E$ which is the union of all the algebraically compact subgroups of $\check{H}^{n}(X)$ with $\check{H}^{n}(X) / E$ an $\aleph_{1}$-free group. We will now show that $E$ is in fact divisible. Since $E$ is torsion free this will make $E$ a vector space over the rationals. Also, once we have shown $E$ to be divisible, then it will be a factor of $H^{m}(X)$ so that

$$
\check{H}^{n}(X) \simeq E \oplus \check{H}^{n}(X) / E \text {. }
$$

Letting $D=E$ and $A=\check{H}^{n}(X) / E^{\prime}$, the corollary will then follow.

Claim. $E$ is divisible.

Proof of Claim. Let $x \in E$ and let $k$ be a positive integer. We will show that $x$ has a $k$ th root in $E$. Let $B$ be an algebraically compact subgroup of $E$ with $x \in B$. Let $B=B_{1} \oplus B_{2}$ where $B_{1}$ is the divisible subgroup of $B$ and $B_{2}$ is a reduced algebraically compact group. According to Theorem 2.8 if $B_{2} \neq 0$, then $B_{2}$ contains a copy of $J_{p}$ or $Z_{p m}$ for some prime $p$. In either case this would contradict our hypotheses in Corollary 3.8. Thus $B_{2}=0$ and $B=B_{1}$ is divisible. Thus $x$ has a $k$ th root in $B$ and hence a $k$ th root in $E$. Thus $E$ is divisible and the claim is proved. This completes the proof of Corollary 3.8 .

3.9. COROLLARY. Let $X$ be an arbitrary n-movable space. Then if $0 \leqslant k$ $\leqslant n$ and $\check{H}^{k}(X)$ is torsion free and does not contain a copy of the p-adic integers $J_{p}$ for some prime $p$, then $\check{H}^{k}(X)$ has the form $\check{H}^{k}(X) \simeq D \oplus A$ where $D$ is a vector space over the rationals and $A$ is $\aleph_{1}$-free.

3.10. ExAMPLE. Let $A$ be a subgroup of the rationals which is not isomorphic to $Z$ or to all of the rationals. Then $A$ is torsion free and the divisible sub. 
group of $A$ is 0 . Clearly $A$ is not $\aleph_{1}$-free since it is countable and not free. Also, $A$ cannot contain a copy of $J_{p}$ since the cardinality of $J_{p}$ is $2^{\mathrm{N}_{0}}$. Thus $A$ cannot be the Čech cohomology group $\check{H}^{k}(X)$ of any movable space $X$ or $n$-movable space with $k \leq n$ by Corollary 3.8 and Corollary 3.9. On the other hand $A$ can be the Ćech cohomology group of a space. Let $\Sigma_{a}=\operatorname{char} A$ be the solenoid whose character group is $A$ and let $X=\Sigma^{k-1} \Sigma_{a}$ be the $(k-1)$-fold suspension of $\Sigma_{a}$. Then $\check{H}^{k}(X) \simeq A$. In [8] Kozlowski and Segal state that $\Sigma^{n} \Sigma_{a}$ is $n$-mov. able. It cannot be $(n+1)$-movable by the above remarks.

4. Paracompacta that are $\mathrm{LC}^{n-1}$. Let $X$ be an arbitrary paracompactum. Then Kozlowski and Segal [9] have shown that if $X$ is $\mathrm{LC}^{n-1}$, then $X$ is $n$-movable. They actually prove a stronger result, but it would not suit the purpose of this paper to state their stronger result here. As a result of [9] we can state the following immediate theorems from the results of the previous section.

4.1. THEOREM. Let $X$ be an $\mathrm{LC}^{n-1}$ paracompactum. Then for $0 \leqslant k \leqslant n$, $\check{H}^{k}(X)$ has a subgroup $E$ which is the union of all the algebraically compact subgroups of $\check{H}^{k}(X)$ and $E$ has the property that $\check{H}^{k}(X) / E$ is an $\aleph_{1}$-free group.

4.2. THEOREM. Let $X$ be an $\mathrm{LC}^{n-1}$ Hausdorff compactum. Then for $0 \leqslant k \leqslant n, \check{H}^{k}(X) /$ Tor $\check{H}^{k}(X)$ is an $\aleph_{1}$-free group.

It is the purpose of this section to show that Theorems 4.1 and 4.2 can be improved for $0 \leqslant k \leqslant n-1$. We then give examples of spaces that are $n$-movable, but not shape equivalent to any $L^{n-1}$ paracompactum.

We now state the improved versions of Theorems 4.1 and 4.2 for $0 \leqslant k$ $\leqslant n-1$.

4.3. THEOREM. Let $X$ be an $\mathrm{LC}^{n-1}$ paracompactum. Then for $0 \leqslant k \leqslant$ $n-1, \check{H}^{k}(X)$ has a subgroup $E$ which is algebraically compact and contains every algebraically compact subgroup of $\check{H}^{k}(X)$. Furthermore, $\check{H}^{k}(X) / E$ is an $\aleph_{1}$-free group.

4.4. THEOREM. Let $X$ be a compact Hausdorff space which is $\mathrm{LC}^{n-1}$. Then for $0 \leqslant k \leqslant n-1, \check{H}^{k}(X)$ is a finitely generated abelian group.

The proof of these theorems is simply that under these circumstances, $\check{H}^{k}(X)=H^{k}(X)$ for $0 \leqslant k<n-1$. We state here the necessary prerequisite result and then proceed to the proofs of these theorems.

4.5. Definition. A space $X$ is said to be homologically locally connected in dimension $n, \mathrm{lc}^{n}$, if for every $x \in X$ and neighborhood $U$ of $x$, there is a neighborhood $V$ of $x$ in $U$ such that $\widetilde{H}_{q}(V) \rightarrow \widetilde{H}_{q}(U)$ is trivial for $q \leqslant n$ where $\widetilde{H}_{q}(X)$ is reduced singular homology with integer coefficients.

The main theorem we need is the following $[16$, Corollary 5, p. 340$]$. 
4.6. THEOREM. If $X$ is a paracompact space which is $1 c^{n}$, then $\check{H}^{k}(X) \simeq$ $H^{k}(X)$ for $0 \leqslant k \leqslant n$.

Proof of Theorem 4.3. We will show that if $X$ is $\mathrm{LC}^{n-1}$, then $X$ is $1 c^{n-1}$. It will then follow from Theorem 4.6 that for $0 \leqslant k \leqslant n-1, \check{H}^{k}(X) \simeq$ $H^{k}(X)$. Thus by Theorem $2.11, \check{H}^{k}(X)$ will have the required structure for $0 \leqslant$ $k \leqslant n-1$. So we need only prove the claim to be finished. The claim seems to be known (see [17] for the locally compact case). We include a proof for completeness.

CLAIM. If $X$ is $\mathrm{LC}^{n-1}$, then $X$ is homologically locally connected in dimension $n-1$.

Proof of Claym. Let $S(X)$ denote the singular semisimplicial complex of $X$ and $|S(X)|$ the geometric realization of $S(X)$ as a CW complex (see [10, Chapter III]). Let $j_{X}:|S(X)| \rightarrow X$ be the natural map [10, Proposition 4.11]. According to Theorem 6.7 of $\left[10\right.$, p. 102] $j_{X}:|S(X)| \rightarrow X$ induces isomorphisms of homotopy groups in all dimensions. If $X$ is a path-connected space, then $j_{X}$ : $|S(X)| \rightarrow X$ also induces isomorphisms between all singular homology groups as well by $\left[16\right.$, Theorem 9, p. 399]. Now we will show that $\operatorname{LC}^{n-1}$ implies $1 c^{n-1}$. Let $x \in X$ and let $U$ be an arbitrary open neighborhood of $x$ in $X$. Since $X$ is $\mathrm{LC}^{0}$ we may assume that $U$ is arcwise connected. Then let

$$
U=U_{n} \supset U_{n-1} \supset \cdots \supset U_{0} \ni x
$$

such that each $U_{i}$ is open and arcwise connected and such that for each $0 \leqslant i \leqslant$ $n-1$ each map $f: S^{i} \rightarrow U_{i}$ extends to a map $F: B^{i+1} \rightarrow U_{i+1}$ as in the definition of locally $i$-connected. Then one can easily show that if $P$ is any polyhedron with $\operatorname{dim} P \leqslant n-1$, and if $f: P \rightarrow U_{0}$, then $f$ is null-homotopic in $U_{n}$. Now consider the diagram

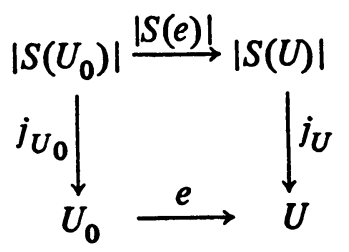

which commutes up to homotopy. Then the diagram

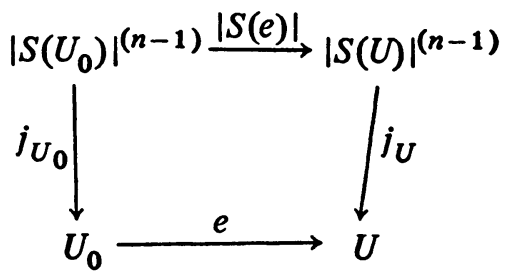

also commutes up to homotopy where $\left|S\left(U_{0}\right)\right|^{(n-1)}$ and $|S(U)|^{(n-1)}$ are the 
$(n-1)$-skeleta of the respective CW complexes. Now the inclusion maps $i$ of $\left|S\left(U_{0}\right)\right|^{(n-1)}$ into $\left|S\left(U_{0}\right)\right|$ and $|S(U)|^{(n-1)}$ into $|S(U)|$ induce isomorphisms of the singular homology groups up to dimension $n-2$ and epimorphisms in dimension $n-1$. Thus we have

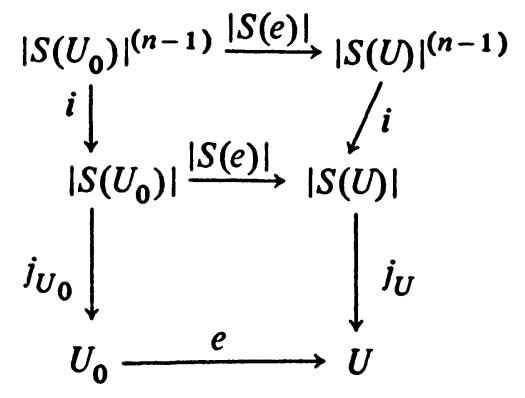

and for $0 \leqslant k \leqslant n-2$, we have

$$
\begin{gathered}
H_{k}\left(\left|S\left(U_{0}\right)\right|^{(n-1)}\right) \stackrel{|S(e)|_{*}}{\longrightarrow} H_{k}\left(|S(U)|^{(n-1)}\right) \\
i_{*}=\left.\simeq\right|_{H_{k}\left(\left|S\left(U_{0}\right)\right|\right)} \stackrel{|S(e)|_{*}}{\longrightarrow} H_{k}(|S(U)|) \\
\left(i_{U_{0}}\right)_{*}=\simeq \downarrow_{H_{k}\left(U_{0}\right)} \stackrel{e_{*}}{\longrightarrow} H_{k}(U)
\end{gathered}
$$

However, $e \circ j_{U_{0}} \circ i$ is null-homotopic in $U$. Thus $\left(e \circ j_{U_{0}} \circ i\right)_{*}: \tilde{H}_{k}\left(\left|S\left(U_{0}\right)\right|^{(n-1)}\right) \rightarrow$ $\widetilde{H}_{k}(U)$ must be the zero homomorphism for $0 \leqslant k \leqslant n-1$. From the above diagram this implies that $e_{*}: \widetilde{H}_{k}\left(U_{0}\right) \rightarrow \widetilde{H}_{k}(U)$ is the zero homomorphism for $0 \leqslant$ $k \leqslant n-2$. For the case that $k=n-1$, one uses the above diagram except that the $i_{*}$ 's are only epimorphisms. One comes to the same conclusion that $e_{*}$ : $\widetilde{H}_{k}\left(U_{0}\right) \rightarrow \widetilde{H}_{k}(U)$ is the zero homomorphism. We have now shown that $X$ is $1 c^{n-1}$ as asserted. The claim is now proved and the Proof of Theorem 4.3 is complete.

Proof OF THEOREM 4.4. Let $X$ be a compact Hausdorff space which is $\mathrm{LC}^{n-1}$. Then according to the Proof of Theorem 4.3, $\check{H}^{k}(X) \simeq H^{k}(X)$ for $0 \leqslant$ $k \leqslant n-1$. Also by the Proof of Theorem 4.3, $X$ is $\operatorname{lc}^{n-1}$. According to Theorem 11 on p. 342 of [16], this implies that $H_{k}(X)$ is finitely generated for $0 \leqslant k$ $\leqslant n-1$. By the Universal Coefficient Theorem for singular cohomology, $H^{k}(X)$ is finitely generated for $0 \leqslant k \leqslant n-1$ also. Thus $\check{H}^{k}(X)$ is finitely generated for $0 \leqslant k \leqslant n-1$ and the proof is complete.

4.7. EXAMPLE. Let $X$ be an $\mathrm{LC}^{n-1}$ Hausdorff compactum. Theorem 4.4 states that $\check{H}^{k}(X)$ is finitely generated for all $0 \leqslant k \leqslant n-1$. Theorem 4.2 states 
that $\check{H}^{n}(X)$ cannot be arbitrary although it does not state that $\check{H}^{n}(X)$ must be finitely generated. Let $S_{\alpha}^{n}$ be the $n$-sphere for all $\alpha \in A$ and let $X=\Pi_{\alpha \in A} S_{\alpha}^{n}$. Then clearly $X$ is an $\mathrm{LC}^{n-1}$ Hausdorff continuum whatever the cardinality of the set $A$. However, $\check{H}^{n}(X) \simeq \bigoplus_{\alpha \in A} Z_{\alpha}$ where each $Z_{\alpha}$ is a copy of the group of integers. This is a free group which agrees with the structure required for this group by Theorem 4.2. However, $\check{H}^{n}(X)$ may have arbitrarily large cardinality by choosing the set $A$ to have appropriately large cardinality. Thus one cannot extend Theorem 4.4 to the case $k=n$.

4.8. EXAMPLE. Theorems 4.3 and 4.4 allow us to give examples of $n$-movable spaces which are not shape equivalent to any $L^{n-1}$ space. Let $X=T^{\omega_{0}}$, the countable infinite product of circles. Then $X$ is movable, hence $n$-movable for every $n$. Now if $X$ were shape equivalent to some compact space which is $\mathrm{LC}^{1}$, then $\check{H}^{1}(X)$ would be finitely generated which it is not. Thus $X$ cannot be shape equivalent to any compact space which is $\mathrm{LC}^{\mathbf{1}}$.

4.9. EXAMPLE. Let $P_{i}$ be a finite connected polyhedron with $H^{2}\left(P_{i}\right) \simeq Z_{i}$. Let $X_{n}$ be the wedge of the $P_{i}$ 's for $i=1, \ldots, n$. Let $f_{n m}: X_{m} \rightarrow X_{n}$ be the map which collapses the extra $P_{i}$ 's $(i=n+1, \ldots, m)$ to the wedge point in $X_{n}$. Let $X$ be the inverse limit of $\left\{X_{n} ; f_{n m} ; n \leqslant m \in N\right\}$. Then $X$ is clearly a movable metric continuum. However, $\check{H}^{2}(X) \simeq \bigoplus_{i=1}^{\infty} Z_{i}$. If $X$ were shape equiv. alent to any paracompactum $Y$ which is $\mathrm{LC}^{2}$, then $H^{2}(X)$ would contain a subgroup $E$ which is algebraically compact such that $\check{H}^{2}(X) / E$ is $\aleph_{1}$-free by Theorem 4.3. However, $\check{H}^{2}(X)$ is pure torsion. Thus $E$ would have to be all of $\check{H}^{2}(X)$. That is, $\bigoplus_{i=1}^{\infty} Z_{i}$ would have to be an algebraically compact group. Now the divisible subgroup of $\bigoplus_{i=1}^{\infty} Z_{i}$ is just zero. Thus $\bigoplus_{i=1}^{\infty} Z_{i}$ would have to be a reduced algebraically compact group which is pure torsion. However, a reduced algebraically compact group which is pure torsion is bounded by Theorem 2.12 . The group $\bigoplus_{i=1}^{\infty} Z_{l}$ is clearly not bounded, a contradiction. Thus $X$ cannot be shape equivalent to any space which is $\mathrm{LC}^{2}$.

The reader can easily create more examples along the lines of Examples 4.7 and 4.8 using Theorems 4.3 and 4.4 .

4.10. EXAMPLE. If $X$ is a compact Hausdorff space which is $L C^{n-1}$, then Theorem 4.4 says that $\check{H}^{k}(X)$ is finitely generated for all $0 \leqslant k \leqslant n-1$. From the Proof of Theorem 4.4 it is clear that $X$ also has the property that $H_{k}(X)$ is finitely generated for $0 \leqslant k \leqslant n-1$. However, $\pi_{k}(X)$ and $\underline{\pi}_{k}(X)$ need not be finitely generated for $0 \leqslant k \leqslant n-1$ even for $X$ a polyhedron. For example, let $X$ be the wedge of $S^{1}$ and $S^{k}$ with $k \geqslant 2$. Then $X$ is LC for all $n$. However, $\underline{\pi}_{k}(X)=\pi_{k}(X)=\bigoplus_{i=1}^{\infty} Z$ is not finitely generated. The structure of $\pi_{k}(X)$ and $\underline{\pi}_{k}(X)$ may be even more complicated as the following example illustrates. Let $f: S^{k} \rightarrow S^{k}$ be a degree 2 map with $k \geqslant 2$. Then let $T_{f}$ be the mapping torus of $f$ formed by identifying the top and bottom of the mapping cylin- 
$\operatorname{der} M_{f}$ of $f$ by the map $f$. This can be done so that $T_{f}$ is a finite polyhedron and thus $\mathrm{LC}^{n}$ for all $n$. Now $\pi_{1}\left(T_{f}\right)=Z$ and the covering space $\widetilde{T}_{f}$ of $T_{f}$ is homeomorphic to an infinite number of $M_{f}$ 's pasted end to end by the map $f$. One can easily verify that $\pi_{k}\left(\widetilde{T}_{f}\right)=Q^{(2)}=\left\{m / 2^{n}: m, n \in Z\right\}$. Thus $\pi_{k}\left(T_{f}\right)=\pi_{k}\left(T_{f}\right)=$ $\pi_{k}\left(\widetilde{T}_{f}\right)$ is a subgroup of the rationals which is neither finitely generated nor free nor isomorphic to the rationals.

\section{REFERENCES}

1. K. Borsuk, On movable compacta, Fund. Math. 66 (1969/70), 137-146. MR 40 \#4925.

2. - On the n-movability, Bull. Acad. Polon. Sci. Sér. Math. Astronom. Phys. 20 (1972), 859-864. MR 47 \#2540.

3. L. Fuchs, Infinite Abelian groups. Vol. I, Pure and Appl. Math., vol. 36, Academic Press, New York and London, 1970. MR 41 \#333.

4. J. Keesling, An algebraic property of the Cech cohomology groups which prevents local connectivity and movability, Trans. Amer. Math. Soc. 190 (1974), 151-162.

5. - On movability and local connectivity, Lecture Notes in Math., vol. 375, Springer-Verlag, New York, 1974, pp. 158-167.

6. Shape theory and compact connected abelian topological groups, Trans. Amer. Math. Soc. 194 (1974), 349-358.

7. - The Cech homology of compact connected abelian topological groups with applications to shape theory, Lecture Notes in Math., vol. 438, Springer-Verlag, New York, 1975, pp. 325-331.

8. G. Kozlowski and J. Segal, n-movable compacta and ANR-systems, Fund. Math. (to appear).

9. Locally well-behaved paracompacta in shape theory (preprint).

10. A. T. Lundell and S. Weingram, The topology of $C W$ complexes, Van Nostrand Reinhold, New York, 1969.

11. S. Mardešić, n-dimensional $\mathrm{LC}^{n-1}$ compacta are movable, Bull. Acad. Polon. Sci. Sér. Sci. Math. Astronom. Phys. 19 (1971), 505-509. MR 46 \#869.

12. - Shapes for topological spaces, General Topology and Appl. 3 (1973), 265-282. MR 48 \#2988.

13. S. Mardešic and J. Segal, Movable compacta and ANR-systems, Bull. Acad. Polon. Sci. Sér. Sci. Math. Astronom. Phys. 18 (1970), 649-654. MR 44 \#1026.

14. - Shapes of compacta and ANR-systems, Fund. Math. 72 (1971), 41-59. MR 45 \#7686.

15. R. H. Overton and J. Segal, $A$ new construction of movable compacta, Glasnik Mat. Ser. III 6 (26) (1971), 361-363. MR 48 \#1157.

16. E. Spanier, Algebraic topology, McGraw-Hill, New York, 1966. MR 35 \#1007.

17. H. B. Griffiths, Local topological invariants. II, Trans. Amer. Math. Soc. 89 (1958), 201-244. MR $21 \# 872$.

DEPARTMENT OF MATHEMATICS, UNIVERSITY OF FLORIDA, GAINESVILLE, FLORIDA 32611 